\title{
Relational Concepts and the Logic of Reciprocity
}

\author{
Yoad Winter \\ Department of Modern Languages and \\ Utrecht Institute of Linguistics OTS, \\ Utrecht University \\ y.winter@uu.nl
}

Research in logical semantics of natural language has extensively studied the functions denoted by expressions like each other, one another or mutually. The common analysis takes such reciprocal expressions to denote $\langle 1,2\rangle$ generalized quantifiers over a given domain $E$, i.e. relations between subsets of $E$ and binary relations over $E$. One of the reoccurring problems has been that reciprocal expressions seem to denote different quantifiers in different sentences. For instance, the reciprocal expression each other means the different quantifiers $Q_{1}$ and $Q_{2} \subseteq \wp(E) \times \wp\left(E^{2}\right)$ in sentences (1) and (2), respectively.

(1) The girls know each other.

$$
Q_{1}=\{\langle A, R\rangle:|A| \geq 2 \wedge \forall x, y \in A[x \neq y \rightarrow R(x, y)]\}
$$

(2) The girls are standing on each other.

$$
Q_{2}=\{\langle A, R\rangle:|A| \geq 2 \wedge \forall x \in A \exists y \in A[x \neq y \wedge(R(x, y) \vee R(y, x))]\}
$$

Following previous work on reciprocals [1|2|3|4 I will suggest that the quantifier that a reciprocal expression denotes takes as parameter certain logical/cognitive semantic properties of relational concepts - intensions of two place predicates. In simple cases this parametrization only involves familiar properties like asymmetry or acyclicity, cf. the relation stand on in (2). However, in more complex cases, also preferences of use and other contextual parameters should be formally described as affecting the logical semantics of reciprocity. The precise formalization of such parameters and their affects on the selection of reciprocal quantifiers will be analyzed, based on recent joint work, logical as well as experimental, with Nir Kerem, Eva Poortman, Sivan Sabato and Naama Friedmann.

\section{References}

1. Dalrymple, M., Kanazawa, M., Kim, Y., Mchombo, S., Peters, S.: Reciprocal expressions and the concept of reciprocity. Linguistics and Philosophy 21, 159-210 (1998)

2. Kerem, N., Friedmann, N., Winter, Y.: Typicality effects and the logic of reciprocity. In: Proceedings of SALT19 (Semantics and Linguistic Theory) (to appear)

3. Sabato, S., Winter, Y.: From semantic restrictions to reciprocal meanings. In: Proceedings of FG-MOL (2005)

4. Winter, Y.: Plural predication and the Strongest Meaning Hypothesis. Journal of Semantics 18, 333-365 (2001) 